\title{
Preparing for the Next Influenza Pandemic
}

\author{
Jonathan A. McCullers, MD \\ Department of Infectious Diseases, St. Jude Children's Research Hospital, 332 N. Lauderdale St., \\ Memphis, TN 38105-2794, Phone: +1 901-495-3486, Fax: +1-901-495-3099, \\ jon.mccullers@stjude.org
}

\section{Abstract}

There are three requirements for an influenza virus to cause a pandemic. It must be antigenically novel, cause severe disease, and transmit easily from human to human. Highly pathogenic avian influenza viruses of the $\mathrm{H} 5 \mathrm{~N} 1$ subtype currently circulating in bird populations in Asia, Africa, and Europe have met 2 of these criteria, and there is significant concern that these viruses will be the cause of the next pandemic. International efforts to prepare for a possible pandemic are underway. Priorities for pandemic planning include surveillance of influenza viruses in wild bird populations and at the avian-human interface, research into factors affecting the pathogenicity of these strains, stockpiling effective antivirals for use as a stopgap until an appropriate vaccine can be developed and distributed, and gaining an improved understanding of the utility of nonpharmaceutical interventions to slow or prevent the spread of these viruses within humans. While considerable progress has been made in recent years towards readying the world for such an event, there is more work to be done. Physicians and hospitals can begin by educating themselves on the problem and developing a pandemic plan for their own practice or organization.

\section{Keywords}

influenza virus; pandemic; vaccine; antivirals; non-pharmaceutical interventions

It has now been more than a decade since highly pathogenic avian influenza (HPAI) viruses of the H5N1 subtype were first found to infect humans. ${ }^{3}$ Although time has passed, the fear that these strains will cause the next pandemic has increased rather than subsided. Estimates of the impact of the next pandemic vary widely depending on assumptions underlying the severity of disease in infected individuals. Morbidity and mortality may not be much higher than in a severe inter-pandemic year, with 314,000 hospitalizations and 89,000 deaths predicted in the United States 21 , or may be staggeringly high in a severe pandemic should a strain similar in capability to the 1918 virus emerge. In the latter scenario as many as 9,900,000

hospitalizations and 1,903,000 deaths 8 could occur in the United States, with between 51 and 81 million deaths worldwide, $96 \%$ of which would occur in the developing world. ${ }^{22}$ Unsurprisingly given these estimates, the economic costs are predicted to be enormous, ranging between $\$ 71.3$ billion and $\$ 166.5$ billion in the United States alone. ${ }^{20,21}$ Advances over the past several decades in our understanding of the evolution and ecology of influenza viruses affords us an unprecedented opportunity at present: the ability to plan and prepare for an infectious disaster that has recurred several times a century throughout history. 1,28 


\section{What is a pandemic?}

Dr. Edwin D. Kilbourne asked this question 30 years ago following the swine influenza outbreak at Fort Dix, New Jersey. ${ }^{14}$ His answer framed the general criteria we use to distinguish pandemic strains from epidemic strains. A pandemic strain must 1) be antigenically novel, 2) cause severe illness, and 3) spread easily through the susceptible population. The H5N1 strains currently infecting humans in Asia and Europe meet 2 of these 3 criteria, lacking only ease of transmission to qualify (Table 1). Each new human infection is an opportunity for the extremely mutable influenza virus to either further adapt to the human host, or reassort with a well-adapted circulating human stain and gain this capability.

Influenza A viruses are subtyped by differences in the major surface glycoproteins hemagglutinin (HA or $\mathrm{H}$ ) and neuraminidase (NA or N). In response to infection with or vaccination against influenza virus, our immune system produces antibodies directed against the HA which bind to the protein and interfere with the virus lifecycle. However, 16 distinct subtypes of HA exist in a wild bird reservoir, primarily in shorebirds and ducks. 6,29 These subtypes are sufficiently distinct that infection with a virus expressing a different HA does not result in antibody-mediated immune recognition and control, allowing full expression of disease in infected individuals and eliminating herd immunity. Thus, spread in humans of an influenza virus with a novel HA allows a high clinical attack rate and the full spectrum of potential disease manifestations.

Although theoretically any of the 14 HA subtypes not currently circulating in humans could generate the next pandemic, 4 subtypes are of particular concern at present. Influenza viruses bearing an H2 HA ceased circulating in humans in 1968, replaced by the H3N2 "Hong Kong" pandemic strain. ${ }^{14}$ Reemergence of this strain would likely trigger a pandemic, as it has already shown this capability in the past, and anyone born after 1968 will be immunologically naïve to the virus. The recent incursions of wholly avian viruses into humans in the last decade provide our other 3 candidates. Most worrisome because of their widespread distribution, capacity to cause severe disease, and evolving host range are HPAI viruses of the H5N1 subtype. $3,9,13$ These strains meet the second requirement, severe disease, as 192 of the 319 laboratory confirmed human cases reported prior to August 2007 were fatal. ${ }^{31}$ However, a recent outbreak caused by an H7N7 strain in the Netherlands ${ }^{7}$ illustrates the point that we cannot focus on a single subtype to the exclusion of others. This strain infected at least 89 persons, one fatally. The third strain of concern, H9N2, has crossed over into humans multiple times in the last decade. ${ }^{2,23}$ Although human disease has been mild with H9N2 in the limited number of cases seen thus far, the virus is widely distributed geographically in domestic poultry and thus has many opportunities to bridge the gap into man. 27

There is only limited evidence so far to support the idea that H5N1 strains are evolving towards a human-adapted strain that can transmit efficiently. Recent H5N1 strains have acquired several mutations thought to be important for generation of a pandemic strain. 5,32 However, they has not yet achieved the breakthrough that will allow them to efficiently transmit and meet all 3 criteria for a pandemic strain. Although human-to-human transmission by close personal contact has been described ${ }^{15}$, sustained chains of transmission or clear evidence of airborne spread have not yet been seen. The virus appears better adapted to the lower respiratory tract of humans than the upper, which may be a factor in its difficulty transmitting thus far. ${ }^{25,26}$ This is probably not the sole reason for poor transmission at present, however, since high viral loads can be detected in the upper respiratory tract of infected patients. ${ }^{4}$

\section{Priorities for pandemic planning}

There are a number of steps that must be taken in order for the world to be prepared for the inevitable next influenza pandemic (Table 2). At the most basic level, knowledge of the 
magnitude and nature of the threat must be understood and monitored with thorough and structured research and surveillance. This starts with programs aimed at understanding the patterns of circulation of influenza viruses in their natural hosts, aquatic waterfowl, and incidental hosts at the human-avian interface such as domestic poultry. ${ }^{16,30}$ Further research into basic questions is needed, such as, "Why are these viruses so lethal? What factors are needed for transmission to humans from birds, or between humans? Can H5N1 strains reassort with currently circulating human strains? Once the pandemic starts, who will be most affected? Will mortality stem from our immune response to the virus, from co-morbid conditions, or from secondary bacterial infections?" We know very little at present about most of these critically important questions.

Much of the public and governmental focus in pandemic preparedness thus far has been on stockpiling antivirals. This is appropriate as a stopgap measure to control the pace of viral spread $^{17}$ or protect key individuals, but is insufficient as a total solution because of cost, distribution, and effectiveness concerns. Unfortunately, the least expensive and most widely available agents, the adamantanes, are not effective against most H5N1 strains. ${ }^{11,19}$ The most popular choice for stockpiling, the neuraminidase inhibitor oseltamivir, has not been highly effective thus far in laboratory confirmed cases of $\mathrm{H} 5 \mathrm{~N} 1$ infection $^{24}$, and resistance has been shown to develop in select treated cases. ${ }^{15}$ Thus, strategies based on prophylaxis of contacts at the start of a pandemic to slow or limit the spread of the pandemic, buying time for development of a vaccine, may be the best option available. ${ }^{18}$

A number of pandemic vaccine candidates are in pre-clinical or clinical development at present, although significant hurdles still exist. ${ }^{12}$ Perhaps the most difficult obstacle is that we will not know what strain or even subtype to make a vaccine against until the pandemic starts. Because of the long production timeline for influenza vaccines (6-10 months), it is unlikely that an effective, well-matched vaccine can be prepared before the first wave of the pandemic virus has circumnavigated the world. Thus, most of the emphasis for planning has to be on mitigation strategies such as antivirals as discussed above, or other measures broadly dubbed "nonpharmaceutical interventions." This includes strategies to prevent or decrease the likelihood of both transmission and acquisition by maintaining personal spacing and decreasing contact between individuals, practicing good hand hygiene, using masks when close contact cannot be avoided, and intervening at the community level by closing schools and discouraging gatherings (Table 2).

\section{Perspective for business}

Every business, especially those in the healthcare field, should have a pandemic plan. A list of useful websites is provided in Table 3. Top priority in the planning process is education of employees about the risks of a pandemic and the measures that will be put into place when it occurs. The most effective and most easily implemented strategy is encouraging good hand hygiene by providing soap and water, or alcohol based hand gels when running water is not available. Next is personal spacing - maintaining inter-personal distances in the workplace of 3-6 feet as this is thought to be the most likely zone within which airborne transmission can occur. ${ }^{10}$ Absenteeism, both planned (work at home programs) and unplanned (personal or family illness) must be accounted for in any pandemic plan, emphasizing the importance of information technology resources and personnel in the "wired" age where work is often not confined to a static workplace. In general, stockpiling of antivirals is not recommended at the level of individual businesses, although pharmacies in healthcare facilities that routinely provide oseltamivir may wish to procure a 10 year supply based on normal usage patterns and rotate this stock by back-filling each year after the influenza season. The Centers for Disease Control has issued interim recommendations on use of masks (Table 3), suggesting that surgical masks are appropriate for situations where close contact or crowds cannot be avoided, and 
reserving N95 respirators for select healthcare applications where aerosolization of virus might be anticipated.

\section{Conclusions}

Our national and international response to the threat of pandemic influenza has been slow to develop, but now appears to be on pace. It is likely that we can be as prepared as is possible within a 5-10 year time frame. Until then, education, planning at the local level, and use of non-pharmaceutical interventions are priorities. Antivirals may be useful in limited scenarios such as initial containment and as prophylaxis of key persons, but are unlikely to represent a panacea. It is unlikely that a sufficient supply of a well-matched, effective vaccine will be available for the first wave of the pandemic if it occurs any time in the next decade. Beyond that, new technologies and promising preclinical candidates may become available, adding to our armamentarium.

\section{Acknowledgments}

Support: NIH AI-66349, American Lebanese-Syrian Associated Charities (ALSAC)

\section{References}

1. Blaser MJ. Pandemics and preparations. J Infect Dis 2006;194:S70-S72. [PubMed: 17163391]

2. Butt KM, Smith GJ, Chen H, et al. Human infection with an avian H9N2 influenza A virus in Hong Kong in 2003. J Clin Microbiol 2005;43:5760-5767. [PubMed: 16272514]

3. De Jong JC, Claas EC, Osterhaus AD, Webster RG, Lim WL. A pandemic warning? Nature 1997;389:554. [PubMed: 9335492]

4. De Jong MD, Simmons CP, Thanh TT, et al. Fatal outcome of human influenza A (H5N1) is associated with high viral load and hypercytokinemia. Nat Med 2006;12:1203-1207. [PubMed: 16964257]

5. Finkelstein DB, Mukatira S, Mehta PK, et al. Persistent Host Markers in Pandemic and H5N1 Influenza Viruses. J Virol. 2007

6. Fouchier RA, Munster V, Wallensten A, et al. Characterization of a novel influenza A virus hemagglutinin subtype (H16) obtained from black-headed gulls. J Virol 2005;79:2814-2822. [PubMed: 15709000]

7. Fouchier RA, Schneeberger PM, Rozendaal FW, et al. Avian influenza A virus (H7N7) associated with human conjunctivitis and a fatal case of acute respiratory distress syndrome. Proc Natl Acad Sci U S A 2004;101:1356-1361. [PubMed: 14745020]

8. Gerberding JL. Pandemic preparedness: pigs, poultry, and people versus plans, products, and practice. J Infect Dis 2006;194:S77-S81. [PubMed: 17163393]

9. Guan Y, Poon LL, Cheung CY, et al. H5N1 influenza: a protean pandemic threat. Proc Natl Acad Sci U S A 2004;101:8156-8161. [PubMed: 15148370]

10. Hall CB. The spread of influenza and other respiratory viruses: complexities and conjectures. Clin Infect Dis 2007;45:353-359. [PubMed: 17599315]

11. Hayden FG, Pavia AT. Antiviral management of seasonal and pandemic influenza. J Infect Dis 2006;194:S119-S126. [PubMed: 17163384]

12. Huber VC, McCullers JA. Vaccines against pandemic influenza: what can be done before the next pandemic? Pediatr Infect Dis J. 2008S1:In press

13. Keawcharoen J, Oraveerakul K, Kuiken T, et al. Avian influenza H5N1 in tigers and leopards. Emerg Infect Dis 2004;10:2189-2191. [PubMed: 15663858]

14. Kilbourne ED. Influenza pandemics in perspective. JAMA 1977;237:1225-1228. [PubMed: 576459]

15. Le QM, Kiso M, Someya K, et al. Avian flu: isolation of drug-resistant H5N1 virus. Nature 2005;437:1108. [PubMed: 16228009]

16. Lipatov AS, Govorkova EA, Webby RJ, et al. Influenza: emergence and control. J Virol 2004;78:8951-8959. [PubMed: 15308692] 
17. Longini IM Jr, Halloran ME, Nizam A, Yang Y. Containing pandemic influenza with antiviral agents. Am J Epidemiol 2004;159:623-633. [PubMed: 15033640]

18. Longini IM IM Jr, Nizam A, Xu S, et al. Containing pandemic influenza at the source. Science 2005;309:1083-1087. [PubMed: 16079251]

19. McCullers JA. Antiviral therapy of influenza. Expert Opin Investig Drugs 2005;14:305-312.

20. Medema JK, Zoellner YF, Ryan J, Palache AM. Modeling pandemic preparedness scenarios: health economic implications of enhanced pandemic vaccine supply. Virus Res 2004;103:9-15. [PubMed: 15163482]

21. Meltzer MI, Cox NJ, Fukuda K. The economic impact of pandemic influenza in the United States: priorities for intervention. Emerg Infect Dis 1999;5:659-671. [PubMed: 10511522]

22. Murray CJ, Lopez AD, Chin B, Feehan D, Hill KH. Estimation of potential global pandemic influenza mortality on the basis of vital registry data from the 1918-20 pandemic: a quantitative analysis. Lancet 2006;368:2211-2218. [PubMed: 17189032]

23. Peiris M, Yuen KY, Leung CW, et al. Human infection with influenza H9N2. Lancet 1999;354:916917. [PubMed: 10489954]

24. Schunemann HJ, Hill SR, Kakad M, et al. WHO Rapid Advice Guidelines for pharmacological management of sporadic human infection with avian influenza A (H5N1) virus. Lancet Infect Dis 2007;7:21-31. [PubMed: 17182341]

25. Shinya K, Ebina M, Yamada S, Ono M, Kasai N, Kawaoka Y. Avian flu: influenza virus receptors in the human airway. Nature 2006;440:435-436. [PubMed: 16554799]

26. Van Riel D, Munster VJ, de Wit E, et al. H5N1 Virus Attachment to Lower Respiratory Tract. Science 2006;312:399. [PubMed: 16556800]

27. Wan H, Perez DR. Amino acid 226 in the hemagglutinin of H9N2 influenza viruses determines cell tropism and replication in human airway epithelial cells. J Virol 2007;81:5181-5191. [PubMed: 17344280]

28. Webby RJ, Webster RG. Are we ready for pandemic influenza? Science 2003;302:1519-1522. [PubMed: 14645836]

29. Webster RG, Bean WJ, Gorman OT, Chambers TM, Kawaoka Y. Evolution and ecology of influenza A viruses. Microbiol Rev 1992;56:152-179. [PubMed: 1579108]

30. Webster RG, Hulse DJ. Microbial adaptation and change: avian influenza. Rev Sci Tech 2004;23:453465. [PubMed: 15702713]

31. World Health Organization. [accessed August 6th, 2007]. Website: http://www.who.int/csr/disease/avian_influenza/country/cases_table_2007_07_25/en/index, html

32. Yamada S, Suzuki Y, Suzuki T, et al. Haemagglutinin mutations responsible for the binding of H5N1 influenza A viruses to human-type receptors. Nature 2006;444:378-382. [PubMed: 17108965] 
Table 1

Requirements for Pandemic Influenz

\begin{tabular}{ll}
\hline A pandemic strain must be... & H5N1 strains... \\
\hline$\ldots$ antigenically novel & $\ldots$ have an H5 HA to which humans \\
& have no pre-existing immunity \\
$\ldots$ virulent enough to cause severe & $\ldots$ have killed $\sim 60 \%$ of all humans \\
disease and some mortality & proven to be infected \\
$\ldots$...ble to transmit easily from & $\ldots$ have shown only limited \\
human to human & transmission by close contact \\
\hline
\end{tabular}


Priorities for Pandemic Planning

\section{Table 2}

Surveillance

Research

Stockpiling antivirals

Pandemic vaccines

Non-pharmaceutical interventions
Wild birds, human-avian interface, human to human transmission

Virulence determinants, capacity to reassort with human strains, requirements for transmission, interactions with the immune system

Capacity for early intervention before vaccine available

Pre- and post-pandemic vaccine strategies

Utility of hand hygiene, masks, social distancing strategies, and community based interventions 
Table 3

\section{Useful website}

World Health Organization avian influenza fact sheet:

http://www.who.int/mediacentre/factsheets/avian_influenza/en/index.html

Avian influenza information for travelers:

http://wwwn.cdc.gov/travel/contentAvianFluInformation.aspx

Preparing workplaces for a pandemic:

http://www.osha.gov/Publications/influenza_pandemic.html

Pandemic planning checklist for hospitals:

http://www.pandemicflu.gov/plan/healthcare/hospitalchecklist.html

United States pandemic plan:

http://www.hhs.gov/pandemicflu/implementationplan/

Guidance on mask use:

http://pandemicflu.gov/plan/community/commaskguidance.pdf 